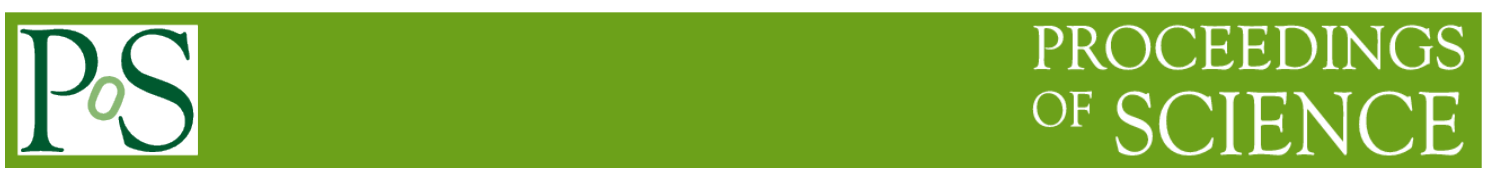

\title{
Overcoming the scattering length limitation in liquid xenon scintillation?
}

\author{
Kai Martens ${ }^{1}$ \\ Kavli-IPMU (WPI), The University of Tokyo \\ Address, Country \\ E-mail: kai.martens@ipmu.jp
}

Having no significant radioactive isotopes and high light yield, liquid xenon is a favorite for low background scintillation detection. With $\sim 40 \mathrm{~cm}$ the Rayleigh scattering length for its scintillation emission at $175 \mathrm{~nm}$ is limited though, presenting a challenge for position sensitive scintillation detection and signal extraction in large single phase detectors. In a 1984 paper it was shown that introducing $200 \mathrm{ppm}$ fluorine into the liquid xenon allows for longer wavelengths emission at $370 \mathrm{~nm}$ and $680 \mathrm{~nm}$ when stimulated with $243 \mathrm{~nm}$ laser light. Since like xenon itself fluorine adds no long lived radioactive isotopes, these longer wavelengths could be an attractive solution to the scattering problem in large low background single phase liquid xenon detectors.

We present a setup designed to verify such longer wavelength scintillation emissions from fluorine doped liquid xenon.

Keywords: dark matter, direct detection, liquid xenon, scintillation

35th International Cosmic Ray Conference - ICRC2017

10-20 July, 2017

Bexco, Busan, Korea

${ }^{1}$ Speaker 


\section{Introduction}

Liquid xenon (LXe) offers high density (shielding), good purification, and - apart from its double beta decay isotope - radiopurity and thus has become the target material of choice for in particular WIMP dark matter searches. Detector dimensions are currently at the one meter scale, but for future detectors at the $\geq 20$ ton scale diameters will grow beyond $2 \mathrm{~m}$, meaning that scintillation light from the well shielded detector center will have to travel more than $1 \mathrm{~m}$ to reach a photomultiplier (PMT) and be recorded. And in pure LXe the Rayleigh scattering length at its scintillation wavelength of $175 \mathrm{~nm}$ is of $\sim 40 \mathrm{~cm}$ by various measurements and calculations - a situation reviewed in [1].

This would mean that scattering dominates the propagation of the scintillation signal in such large detectors: a quick simulation with a conservative $50 \mathrm{~cm}$ Rayleigh scattering length and no attenuation of the signal as it Rayleigh-scatters its way to the surface of an idealized spherical detector is summarized in the following table:

\begin{tabular}{|l|l|l|}
\hline $\begin{array}{l}\text { Spherical detector radius } \\
{[\mathrm{cm}]}\end{array}$ & $\begin{array}{l}\text { average number of scatters } \\
\text { before reaching surface }\end{array}$ & $\begin{array}{l}\text { average length of travel to } \\
\text { surface }[\mathrm{cm}]\end{array}$ \\
\hline 40 & 1.07 & 54 \\
\hline 1.25 & 5.25 & 260 \\
\hline
\end{tabular}

The short wavelength of the scintillation emission is the main driver of this conundrum. Already in [1] the authors point out that the way around this problem is to change the emission wavelength by adding a soluble component that emits at a longer wavelength - as Xe does in liquid argon, the only two component system for they compare measurement and calculation.

In their discussion section they point to a 1984 paper [2] that investigates the eximer emissions from fluoride dissolved in liquid noble gases, and in particular to that papers results on the addition of fluorine to LXe. As the authors of [1] point out, fluorine has only one stable isotope, and thus would not spoil the radiopurity of a LXe target. As [2] uses laser light to excite the emission, the authors of [1] conclude that "the efficiency of the energy transfer from Xe to mixed eximers and the dependence of this process on concentration of the solute must be explored to assess whether the addition of fluorine is a viable option." This is what the setup below aims to do.

\section{Scintillation emission in LXe}

The standard scintillation emission in pure LXe at $175 \mathrm{~nm}$ originates from a $\mathrm{Xe}_{2} *$ dimer [3]. This is the only emission that can be exploited for scintillation detection in LXe, and is routinely used by both, single and two phase LXe detectors. As detailed in [2] adding fluorine to the LXe creates the the opportunity for more complex eximers to be formed and to decay. [2] reports the observation of a line at $371 \mathrm{~nm}$ attributed to an $\mathrm{Xe}_{2} \mathrm{~F}^{*}$ trimer, at $560 \mathrm{~nm}$ attributed to an $\mathrm{XeF}^{*}$ dimer, and at $680 \mathrm{~nm}$ attributed to an $\mathrm{Xe}_{2} \mathrm{~F}^{*}$ trimer again. All these emissions were observed after stimulation with a laser beam at a wavelength significantly longer than $175 \mathrm{~nm}$. 
What will happen if the stimulation is by a charged particle moving through the fluorine doped LXe? Will the "traditional" $\mathrm{Xe}_{2}{ }^{*}$ dimer channel harvest most of the excitation and reduce emissions by other eximers to unusably faint signals? Will any of the other eximers pull that trick and move the scintillation emission to a longer wavelength entirely? Or will there be a balance of emissions at various wavelength from a mix of the available eximer states? If this latter case is found to be how nature does things, then the exciting possibility arises that the relative intensity from competing de-excitation channels might carry information about the density of energy deposit at the origin - in which case electron and nuclear recoil might become separable from the scintillation signature alone: Particle ID or background rejection might be possible. Depending on the timing characteristics of the various excimers' decays this could then possibly even be done with just one type of PMT with a sensitivity stretching over at least the relevant wavelengths.

The first question to be answered though is: How significant is the overall scintillation output of fluorine doped LXe, and how does it depend on the concentration of fluorine in LXe. In the following we will discuss a very basic setup that is designed to quickly get an answer to this most basic question so that funding for an improved setup can be obtained if first results are promising. The setup described below is tested and ready for use; due to regulations regarding the use of fluorine in Japan it will need to be shipped to Chicago though to make real measurements, where the University of Illinois at Chicago and in particular the laboratory where the original measurements for the 1984 paper from the group led by Prof. Rhodes were made have kindly agreed to host this effort. That laboratory is still set up for work with fluorine at the concentrations required here.

\section{The experimental setup}

With no dedicated funding available and the "success" uncertain at best this first setup is as simple as it can possibly be:

\section{LXe cell and PMTs}

The body of the cell is essentially a doulbe sided, $1 \mathrm{~cm}$ wide ICF 34 ring with a $1 / 8$ th inch VCR radial port capped by two $1 \mathrm{~mm}$ thick MgF2 windows, one on either side in its own ICF 34 flange. Both windows are alike, and provide a circular viewport of $1 \mathrm{~cm}$ diameter into that ICF sandwich of the two windows capping the ring with its port.

On the inside of that cell sits a PTFE tube machined to minimize dead volume beyond its central $1 \mathrm{~cm}$ diameter bore that connects the two viewports. A $2 \mathrm{~mm}$ drilled hole connecting to a v-shaped channel on the outside of this PTFE filler allows both gas and liquid to enter this central scintillation volume and connects it to the $1 / 8$ th inch radial port. This PTFE filler almost extends to touch the MgF2 windows, but seen from the outside stays behind their seating and seal. Its inner diameter is matched to that of the windows, so that the high reflectivity of the PTFE can help to couple light into the vacuum outside. The PMTs used in this setup, a 
Hamamatsu R9875P with sensitivity for both the $175 \mathrm{~nm}$ and the $371 \mathrm{~nm}$ lines, and a Hamamatsu R7600U with sensitivity for both the $560 \mathrm{~nm}$ and the $680 \mathrm{~nm}$ lines are not suitable for use at cryogenic temperatures and therefore unfortunately cannot be optically coupled to the LXe cell's MgF2 windows. Via coincidence with a standard XMASS R10789 PMT on one side of this cell and the R9875P on the other the both looking at 60Co induced scintillation emission from pure LXe in the cell a reference measurement and proof of the detectability of scintillation signals from this cell at least with pure LXe has been established.

\section{Conclusion}

The list of reasons why xenon is a favorite target material for dark matter (DM) and other rare event searches are well known and advertised. Adding fluorine will not affect the central radiopurity argument for xenon but may offer longer wavelength emission that can traverse larger distances and therefore is more amenable to extracting signal from bigger the bigger sized detectors of the future. If luck should have it, there may yet be a way to recover particle ID from the relative contributions of the different emission lines if they indeed all contribute to the scintillation signal and can be separated by timing signature or, exploiting differences in their respective wavelengths, by detection in specialized subsets of photosensors. Of course the addition of fluorine will not allow to drift electrons any more, and two phase detectors cannot use this solution to shift the wavelength of the scintillation emission. Unless of course we start drifting ions.

\section{Acknowledgments}

The author wishes to thank his colleagues at the XMASS experiment for their advice and many a fruitful discussion, as well as for the temporary use of an XMASS PMT and Xe gas during preparations in Kamioka. Special thanks go to Prof. emeritus Charles Rhodes at UIC in Chicago for introducing me to his department and allowing the use of his lab for proceeding with the experiment. We gratefully acknowledge the cooperation of the Kamioka Mining and Smelting Company. Travel to Chicago was supported by JSPS Kakenhi grant number 26104004.

\section{References}

[1] G.M. Seidel et al., Rayleigh scattering in rare-gas liquids, NIM A489 (2002) 189-194.

[2] H. Jara et al., Optical properties of rare-gas fluoride dimers and trimers dissolved in liquid rare gases, Phys. Rev. B 30 (1984) 1.

[3] A. Hitachi, Properties of liquid xenon scintillation for dark matter searches, Astropart. Phys. 24 (2005) 247-256. 